\title{
MEASUREMENTS OF THE STEADY CURRENTS OUTSIDE THE SURF ZONE
}

\author{
Pietro Scandura ${ }^{1}$ Erminia Capodicasa ${ }^{1}$ and Enrico Foti ${ }^{1}$
}

\begin{abstract}
The results of an experimental study concerning with the measurements of the steady current induced by sea waves approaching the coast are reported. The experiments have been performed in a large wave flume in order to minimize the scale effects. The measurements have been carried out at four different stations along the wave flume by using Acoustic Doppler Velocimeters. The results show that the mean velocity profiles are significantly influenced by the wave period. In particular, when the period reduces the mean velocity gradient close to the wave trough increases. The trend of the velocity profiles is different from that predicted by the theory and mostly reported by other experiments. However, experimental results are reported in literature which are in a qualitative agreement with the present ones.
\end{abstract}

Keywords: sea waves; steady currents; velocity measurements

\section{Introduction}

A deep knowledge of the complex hydrodynamics induced by sea waves propagating towards the coast is important for several reasons and in particular in order to formulate reliable models to predict the sediment transport and the diffusion of pollutants. In this context one of the most important characteristics of the flow is the steady velocity generated by the nonlinearity of the waves themselves. As concerns the global flow produced by the steady velocity, for a sea wave normally approaching the coast, the onshore flux produced between the trough and the crest, because of mass conservation, is balanced by a backward flow also denoted as 'undertow'. Even though the steady velocity is weak compared to the oscillating component, it plays an important role in the transport processes because of its persistency.

The distribution along the water depth of the mean velocity is influenced by the steady streaming generated in the bottom boundary layer and to a lesser extent by the steady vorticity generated in the free surface boundary layer. The existence of a steady streaming in the bottom boundary layer was observed experimentally by Bagnold (1947) and then it was explained theoretically by Longuet-Higgins (1953) for the case of a laminar flow by introducing the effect of the viscosity which had been neglected in the classical wave theory of Stokes (1947). The main effect of the viscosity is to induce a mean Reynolds stress in the boundary layer the gradient of which in turn drives a mean velocity.

The experiments of Russel and Osorio (1958) confirmed the theoretical results of Longuet-Higgins (1953) but further experimental studies such as those of Brebner et al. (1966) and Bijker (1974), showed that the steady streaming in the bottom boundary layer can be smaller than that predicted theoretically when the bottom boundary layer is not laminar.

In the previous experiments the prediction of the theory could be not fully correct because of the presence of asymmetric waves and of turbulence in the bottom boundary layer. Indeed, it has been shown that when the waves are asymmetric, the different intensity of the turbulence during the seawards and landwards parts of the wave period causes an offshore directed steady streaming (Scandura 2007, Holmedal and Myrhaugh 2009).

How the steady streaming affects the distribution of the mean velocity along the water depth has been explained by Longuet-Higgins (1953) and also by Putrevu and Svendsen (1993) by assuming the shallow water approximation.

Although several laboratory experiments have been carried out in order to gain insights about the the backward flow produced by the waves, it seems that up to now experiments with waves characterized by a turbulent bottom boundary layer have not been performed yet. In this regard, the experimental difficulty is due to the fact that in order to have a turbulent boundary layer it is necessary to generate large waves characterized by dimensions that exceed the capabilities of most experimental facilities. In some experiments, velocity fluctuations reproducing those observed at high Reynolds number have been induced by covering the bed by gravel (Sleath 1984). However, the macro-roughness due to the gravel generates large vortices which in a small wave flume could affect the flow much more than in the field. For such a reason the present experimental investigation has been carried out in a large wave flume where in most of the wave conditions the boundary layer is in the turbulent regime.

\footnotetext{
${ }^{1}$ Department of Civil and Environmental Engineering, University of Catania, Viale A. Doria 6, Catania, Italy, 95125
} 


\section{The experimental facility}

The experiments have been carried out in the CIEM large wave flume at the 'Universitàt Politecnica de Catalunya' (UPC) in Spain. A sketch of the wave flume is shown in Fig. 1. The flume is $100 \mathrm{~m}$ long, $3 \mathrm{~m}$ wide and $5 \mathrm{~m}$ deep. The bottom is made up of sand with $\mathrm{d}_{50}$ equal to $0.25 \mathrm{~mm}$. The origin of the reference system is located in the middle of the stroke of the wave paddle and lies on the rigid bottom of the wave flume. The $x$ axis is positive in the onshore direction while the $y$ axis is directed vertically and points upwards. In the following figures the distances from the sandy bottom, measured along the vertical direction, are denoted by $y_{\mathrm{b}}$. At about $x=44 \mathrm{~m}$ a beach having a slope of 1:15 begins. The measurements have been carried out along the water depth at four different stations $(x=33.93,44.11,55.33,61.6 \mathrm{~m})$ shown in Fig. 1.

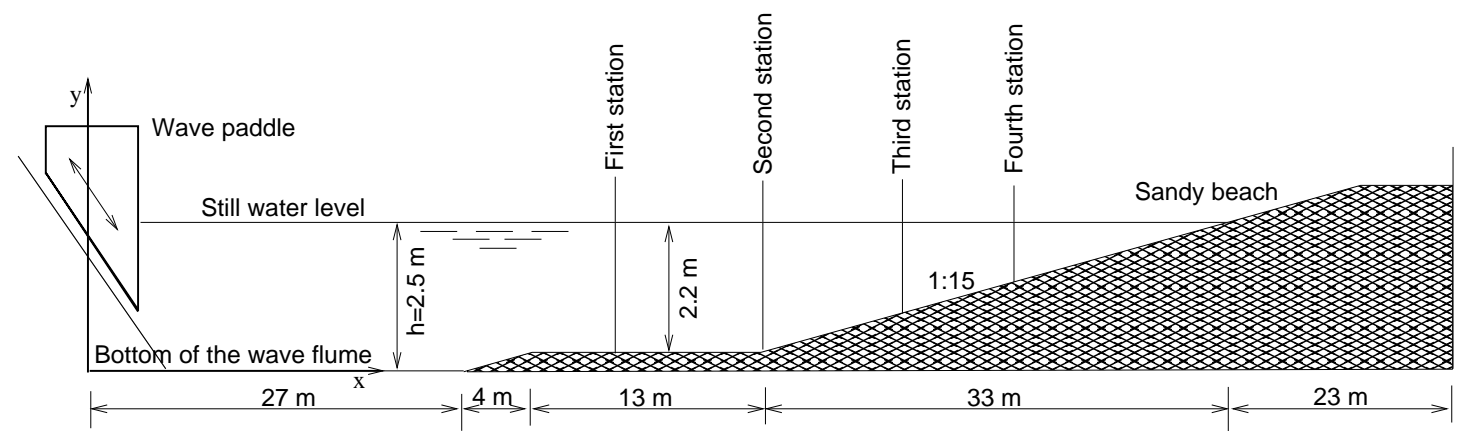

Figure 1. Longitudinal section of the CIEM large wave flume at the Universitàt Politecnica de Catalunya (Spain).

The water depth was equal to $2.5 \mathrm{~m}$ in all the experiments. The velocity has been measured by eight Acoustic Doppler Velocimeters deployed along the water depth. The water levels have been measured by using 13 wave gauges. The position of the bottom was detected by a mechanical profiler placed on a carriage which allows the bottom profile along the middle of the channel to be measured.

Fifteen different kind of regular waves have been produced in each station by combining five different wave heights $H(H=0.2,0.3,0.4,0.5,0.6 \mathrm{~m})$ with three different wave periods $T(T=3,4.25$, $5.5 \mathrm{~s})$. The waves have been generated according to the Stokes linear wave theory. However, during the propagation the waves became asymmetric, especially those which were characterized by a large wavelength.

During the experiments three-dimensional ripples were observed on the sandy bottom which were characterized in average by a wavelength of $20 \mathrm{~cm}$ and a height of 3-4 cm. However, there was a large variability of these quantities in the channel.

\section{Experimental results}

In order to gain insights about the kind of waves that have been generated, in Fig $2 \mathrm{a}$ the time development of the water level and of the velocity measured at $1.22 \mathrm{~m}$ from the sandy bottom in the first station, for $H=0.5 \mathrm{~m}$ and $T=5.5 \mathrm{~s}$, are reported and compared with the irrotational wave theory over a constant depth at the third order of approximation.
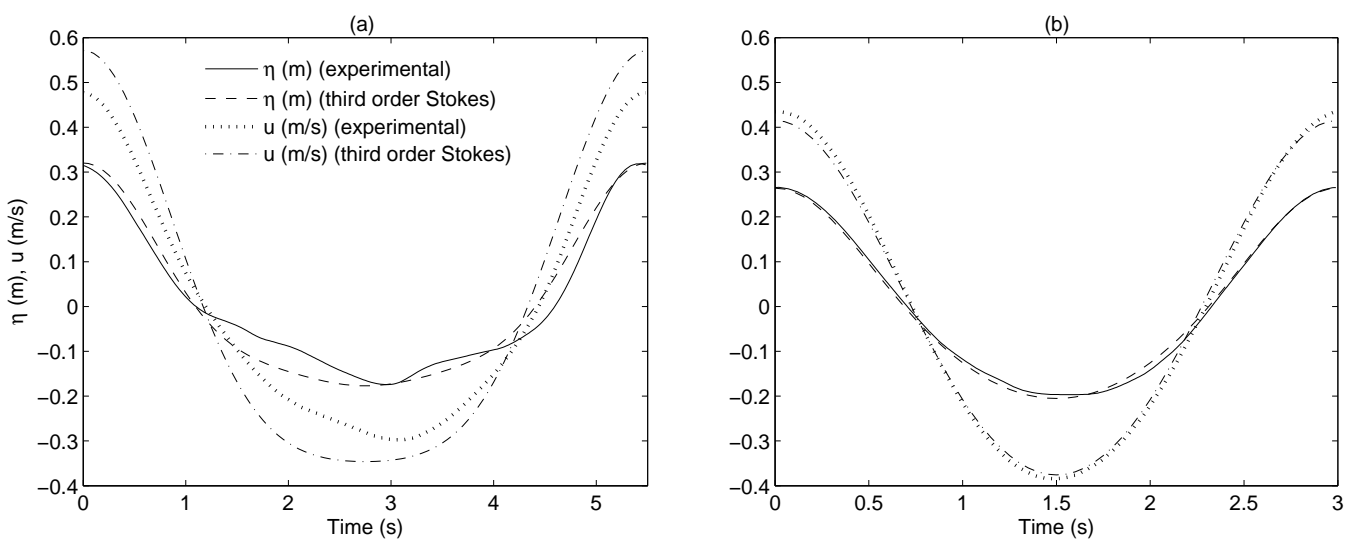

Figure 2. Time development of the water level $\eta$ and of the mean velocity $u$ at $1.22 \mathrm{~m}$ from the bottom. (a) $H=0.5 \mathrm{~m}, T=5.5 \mathrm{~s}$; (b) $H=0.5 \mathrm{~m}, T=3.0 \mathrm{~s}$. 
The experimental results are phase averaged over 450 wave periods. We observe that a discrepancy between experiments and theory is present especially as regards the velocity. The discrepancy is due to the strong interaction of these long waves with the bottom which causes the generation of harmonic components which are out of phase among them and therefore they cannot be reproduced by the Stokes wave theory. The agreement between theory and experiments improves significantly when the period reduces as it can be observed in Fig. $2 \mathrm{~b}$, where the results for $H=0.5 \mathrm{~m}$ and $T=3 \mathrm{~s}$ are reported.

We also observe that the waves are asymmetric as the wave amplitude at the crest is larger than that at the trough. Generally, the asymmetry increases when the wave period and/or the wave height increases.

In Fig. 3a, where the mean velocity profiles for $T=5.5 \mathrm{~s}$ are shown, it can be observed that when $H$ increases the velocity tends to be more negative both close to the wave trough and close to the bottom, while in the middle of the water column it does not appear to be significantly affected by the wave height.
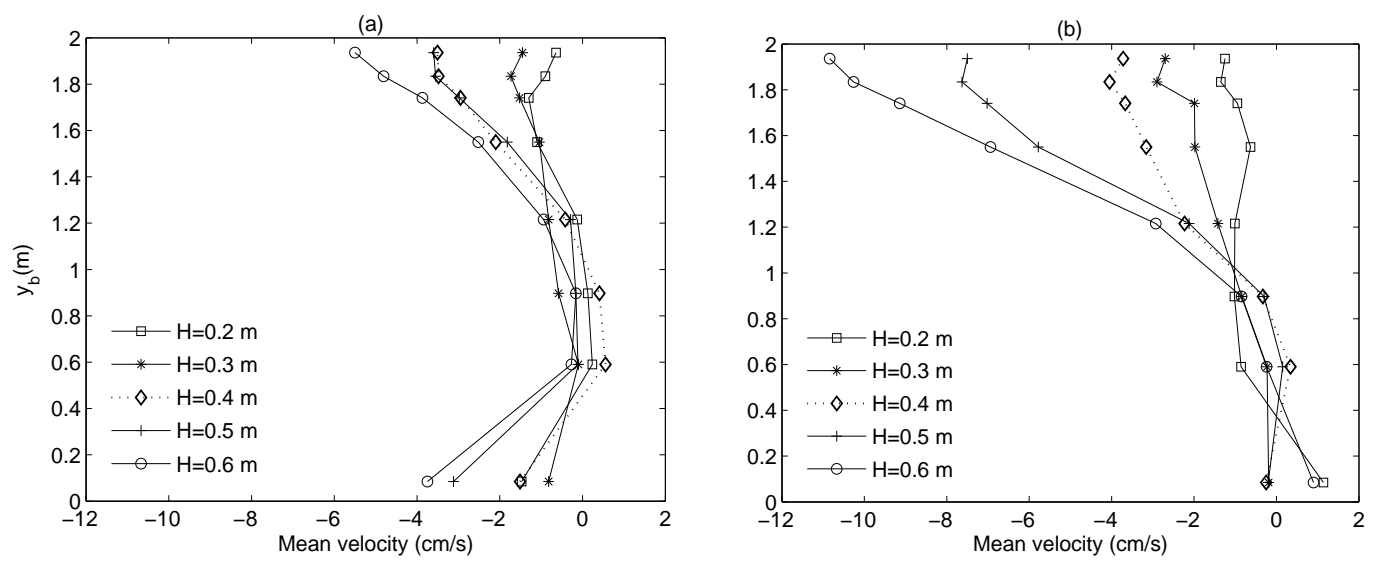

Figure 3. Mean velocity profiles in the first station for (a) $T=5.5 \mathrm{~s}$, (b) $T=3 \mathrm{~s}$.

The tendency of the mean velocity to take negative values close to the trough increases when the wave period reduces, as it can be observed by comparing Fig $3 \mathrm{a}$ with Fig $3 \mathrm{~b}$ where the results for $T=3 \mathrm{~s}$ are reported. At the bottom we observe that when the period reduces the velocity tends to be more positive.

It is interesting to highlight that the trend of these mean velocity profiles is qualitatively different from that reported by previous works on the subject. In particular, the diffusion solution of LonguetHiggins (1953) and the experimental results of Hwung and Lin (1990) and those of Nadaoka and Kondoh (1982) show that the mean velocity profiles have the convexity turned towards offshore while present results show just the opposite. This issue will be reconsidered in the following.

In Fig. 4 and in Fig. 5 the mean velocity profiles measured in the different stations along the channel are reported for $H=0.5 \mathrm{~m}$ and $T=5.5 \mathrm{~s}$ and $T=3 \mathrm{~s}$ respectively. When the velocity profiles show a discontinuity close to the wave trough, this is due to the fact that the ADV probe, placed in the most elevated position, measured very close to the wave trough or just above it where the gradient of the mean velocity is very high (Hwung and Lin 1990). In the following we will discuss the velocity profiles below the wave trough only.

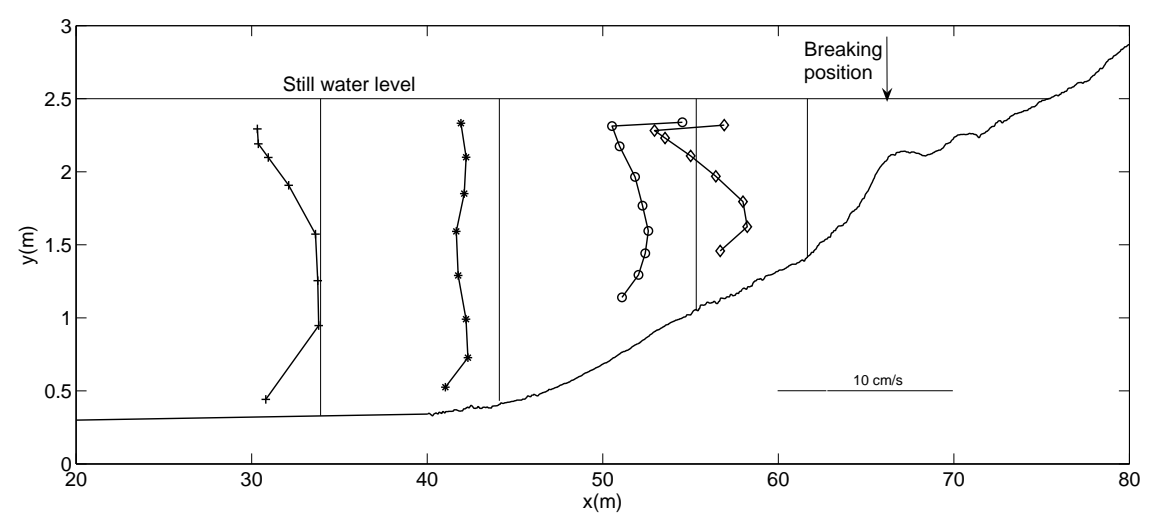

Figure 4. Mean velocity profiles along the channel for $H=0.5 \mathrm{~m}$ and $T=5.5 \mathrm{~s}$. 


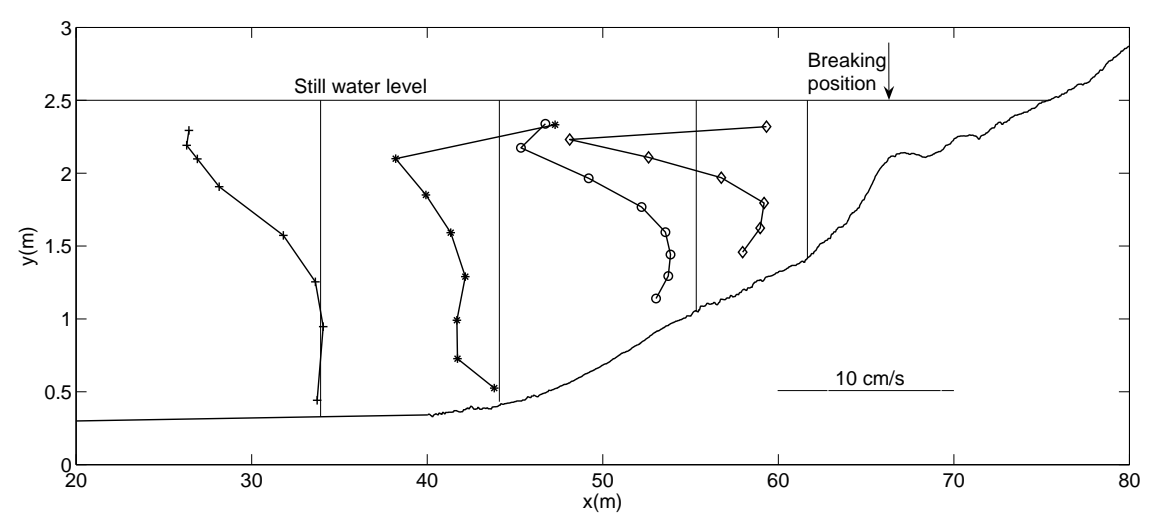

Figure 5. Mean velocity profiles along the channel for $H=0.5 \mathrm{~m}$ and $T=3.0 \mathrm{~s}$.

During the experiments the bottom profile underwent important modifications close to the bar only. In the rest of the channel, and in particular offshore the bar, the modifications were mainly due to small scale bedforms. In Figs. 4 and 5 the profile of the bottom is the most representative among those that have been observed during the experiments. We note that in shallow depth the velocity and its gradient have large values both close to the trough and close to the bottom. The velocity profile close to the toe of the beach for the larger period is an exception as it is rather constant along the depth. In the first station for $T=3 \mathrm{~s}$ the mean flow close to the bottom appears to be weakly affected by the waves.

In Fig. 6a the time mean velocity profiles for $T=5.5 \mathrm{~s}$ in the third station, which is placed along the sloping bottom, is reported. We can observe that when the wave height increases the mean velocity increases along the water depth much more uniformly than in the first station (see Fig. 3a). In Fig. 6b the mean velocity profiles in the third station for $T=3 \mathrm{~s}$ are shown. By comparing these results with those of Fig. 6a, it can be observed that when the period reduces the velocity at the bottom becomes more positive, while close to the wave trough it becomes more negative. This behavior leads to an increase of the variability of the mean velocity along the depth when the period reduces. Such result is quite general as it has been observed even in Fig. 3 for the first station.
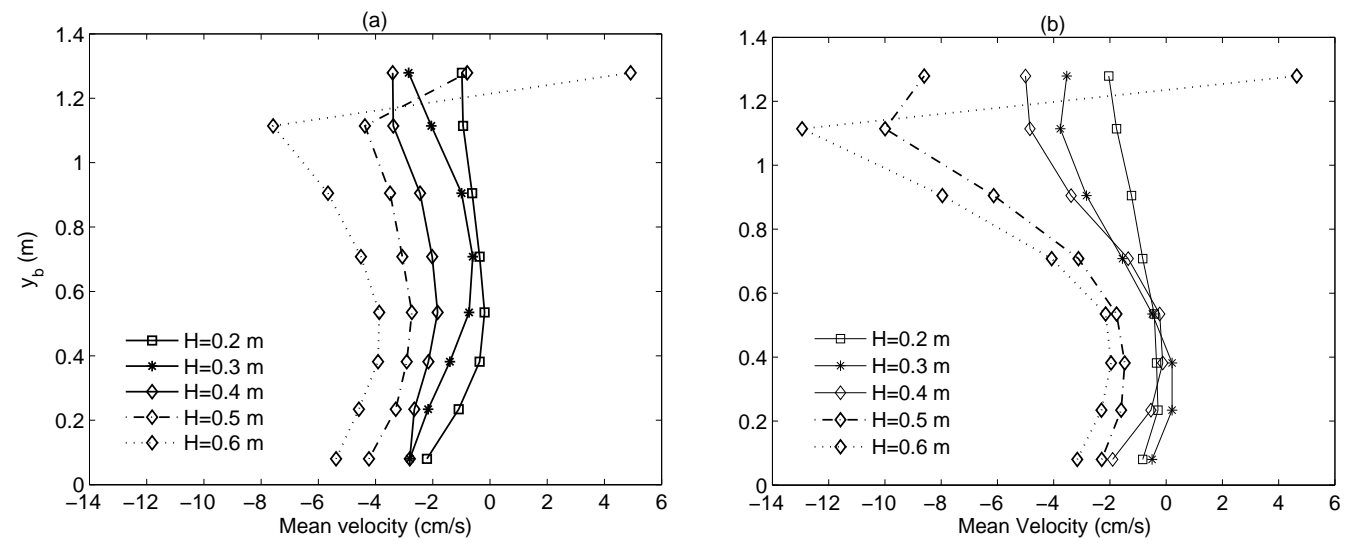

Figure 6. Mean velocity profiles in the third station for (a) $T=5.5 \mathrm{~s}$, (b) $T=3 \mathrm{~s}$.

Up to now we have considered mean velocity profiles obtained by averaging over a large number of wave periods in order to reduce the sampling variability. In order to analyze the evolution of the mean velocity profiles during the experiments, it is necessary to average the velocity over few wave periods. In particular, the results shown in Fig. 7 have been obtained by averaging during 3 periods at different stages of the experiments. We highlight that not all the experiments that have been carried out are appropriate for such kind of analysis, as very often during the initial stage of an experiment the ADV signal were affected by spikes. Such spikes were due to a not sufficient concentration of particles in suspension necessary to produce an adequate reflection of the acoustic beam. Usually the spikes diminish and then disappear during the experiments thanks to fine particles brought in suspension by the tangential stresses acting on the bottom.

In Fig. 7 the trend of the mean velocity at three different times during the experiments in the third station are reported. Although the mean velocity profiles at the early stage of flow development show 
the convexity turned towards offshore, in all the cases after the flow develops the curvature is just the opposite. We also observe that at the beginning the mean velocity is rather constant, which witnesses that the mean flow is close to be irrotational. This result is expected as the vorticity produced on the boundaries requires a finite amount of time in order to diffuse in the interior of the fluid. Finally, it can be observed that at the beginning the flux due to the mean velocities is different from that established at later stages.
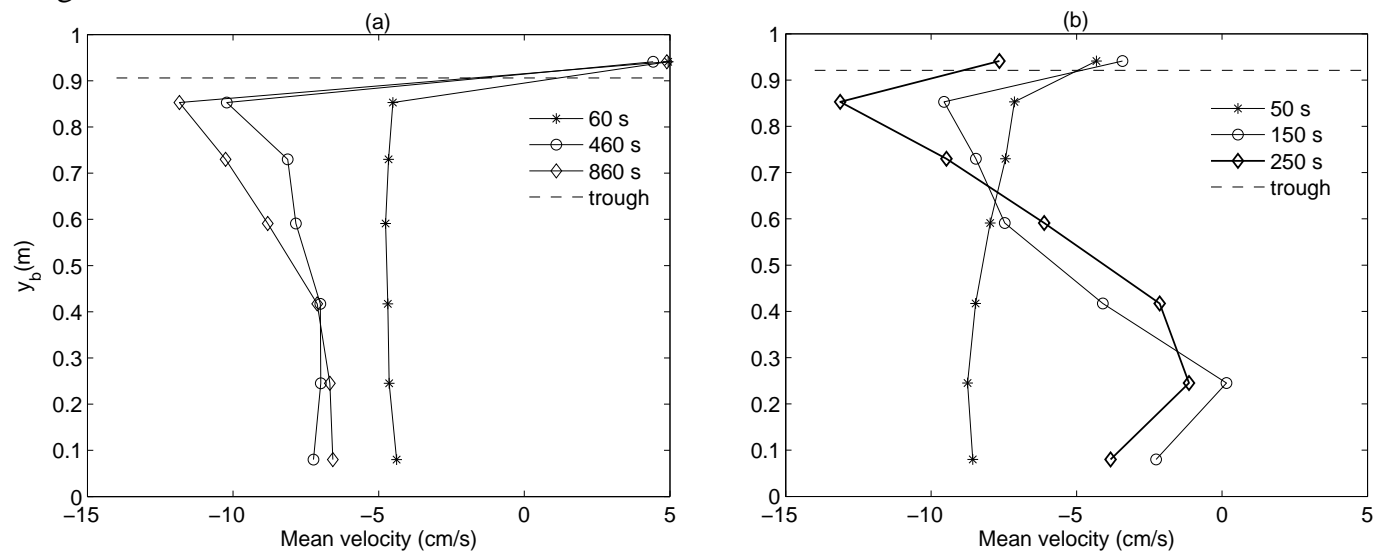

Figure 7. Mean velocity profiles in the fourth station at different stages during the experiments for (a) $T=5.5$, (b) $T=3 \mathrm{~s}$.

An explanation of the offshore mean velocity observed close to the seabed, which is opposite to that determined by the theory of Longuet-Higgins (1953), can be given by taking into account that the bottom boundary layer is turbulent and the waves are asymmetric. Indeed, in such a case, in the bottom boundary layer, apart the contribution to the mean Reynolds stress due to the variability of the flow along the direction of wave propagation (Longuet-Higgins 1953), there is also a contribution due to the turbulence. This second contribution arises because of the different intensity of the turbulence between the seawards and landwards parts of the wave period when the wave is asymmetric. While the first contribution generates a steady streaming directed onshore, the second one generates a steady streaming directed offshore (Scandura, 2007; Holmedal and Myrhaugh, 2009). In order to evaluate the effect of the wave asymmetry, we introduce the wave asymmetry defined as

$$
A_{s}=\frac{\left|U_{c}\right|-\left|U_{t}\right|}{\left|U_{c}\right|+\left|U_{t}\right|},
$$

where $U_{\mathrm{c}}$ and $U_{\mathrm{t}}$ are the velocities under the wave crest and under the wave trough respectively, measured by the ADV probe closest to the bottom $(8 \mathrm{~cm}$ from the bottom), and the dimensionless mean velocity $U_{\mathrm{s}}$,

$$
U_{s}=2 \frac{U_{m}}{\left|U_{c}\right|+\left|U_{t}\right|}
$$

where $U_{\mathrm{m}}$ is the dimensional mean velocity measured by the previous ADV probe. In Fig. 8, where $U_{\mathrm{s}}$ is reported versus the asymmetry $A_{\mathrm{s}}$, although the data are rather scattered, it can be observed that when the asymmetry increases the mean velocity becomes more negative. For small values of the asymmetry, the intensity of the turbulence during the seawards and the landwards parts of the wave period is similar, therefore the mechanism described by Longuet-Higgins (1953) prevails and the steady streaming can be directed onshore. 


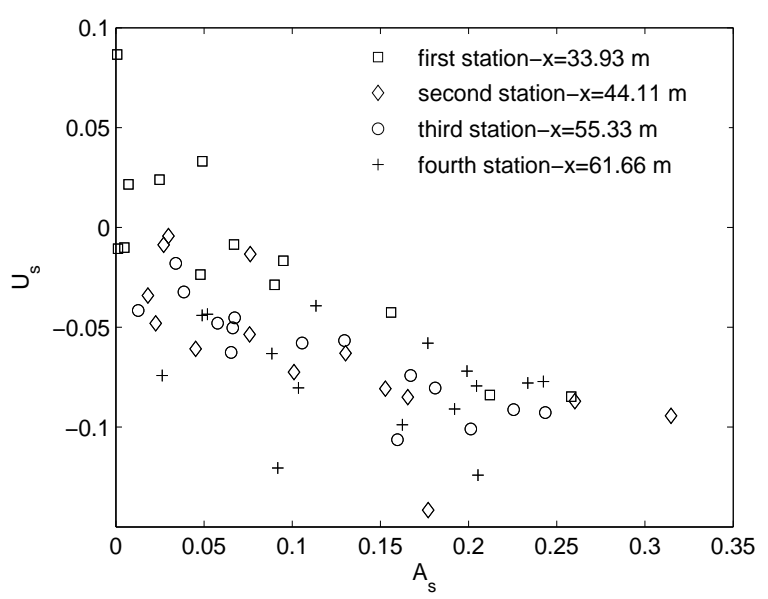

Figure 8. Mean dimensionless velocity close to the bottom versus the wave asymmetry $\boldsymbol{A}_{\mathrm{s}}$.

The previous analysis is pertinent to the flow near the bed and does not provide an explanation about the trend of the mean velocity along the entire water depth.

It is known that the mean Lagrangian velocity $U_{\mathrm{L}}$ of the fluid particles, at the second order of approximation in the wave steepness, can be evaluated by adding the Stokes drift $U_{\mathrm{St}}$ to the mean Eulerian velocity $U_{\mathrm{E}}$ as follows:

$$
U_{L}=U_{S t}+U_{E}
$$

Recently, Monismith et al. (2007) analyzed several laboratory experiments concerning with the interaction between waves and currents in which the mean Lagrangian velocity of the current appears to be not affected by the superimposition of waves. In other words, the findings of Monismith et al. (2007) show that when a sea wave is superimposed to a steady current, a changing in the mean Eulerian velocity takes place such that it cancels, in Eq. (3), the Stokes drift due to the waves. In the present case, since prior to running the waves we do not have any steady current, the mean Eulerian velocity should be opposite to the Stokes drift at each depth, therefore

$$
U_{\mathrm{E}}=-U_{\mathrm{St}} .
$$

The author where not able to provide an explanation of this results as the theory shows that the Eulerian mean velocity balances the Stokes drift only in an integral sense. The authors highlighted that for $k h \approx 1$ the previous result does not hold while it holds for $k h$ significantly larger than one, where $k$ is the wavenumber and $h$ is the water depth.

Although the present experiments are characterized by values of $k h$ smaller than 1.18 , a comparison between the Stokes drift and the mean Eulerian velocity has been performed in order to check to what extend present results agree with Eq. 4.

Then, first we have evaluated the Stokes drift according to the following formula:

$$
U_{\mathrm{St}}=\overline{\frac{\partial u}{\partial x} \int u d t}+\overline{\frac{\partial u}{\partial y} \int v d t}
$$

where the bar denotes a time average, $u$ is the velocity component in the direction of wave propagation and $v$ the velocity component along the vertical direction. The partial derivative of $u$ with respect to $x$ has been computed by using the continuity equation. The derivatives have been evaluated numerically by the finite difference approximations in the middle between two ADV probes. In Fig. 9 the trend of the mean Eulerian velocity and of the Stokes drift evaluated by using the velocity measurements, along with the theoretical Stokes drift computed by using the formula valid for irrotational waves propagating over a constant depth (Dean and Dalrymple 1992) are shown. 
(a)

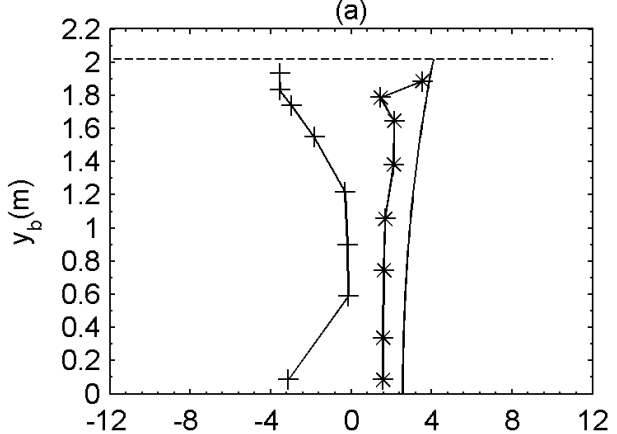

(c)

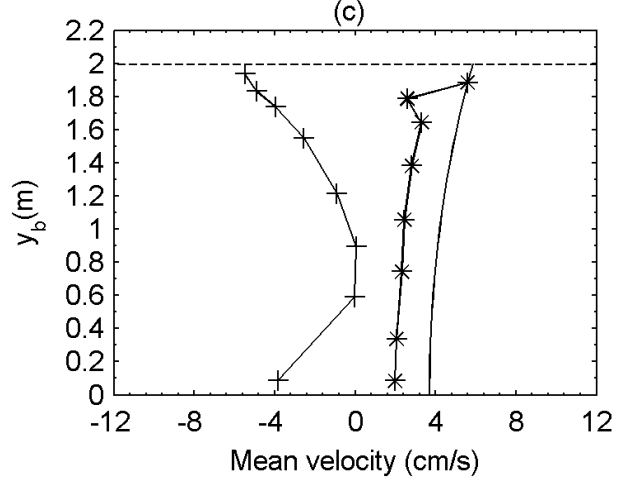

(b)

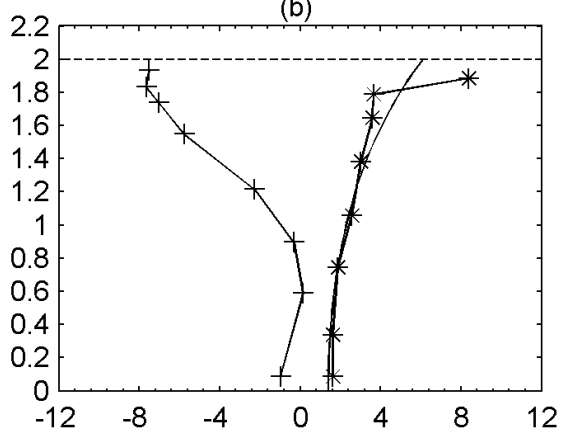

(d)

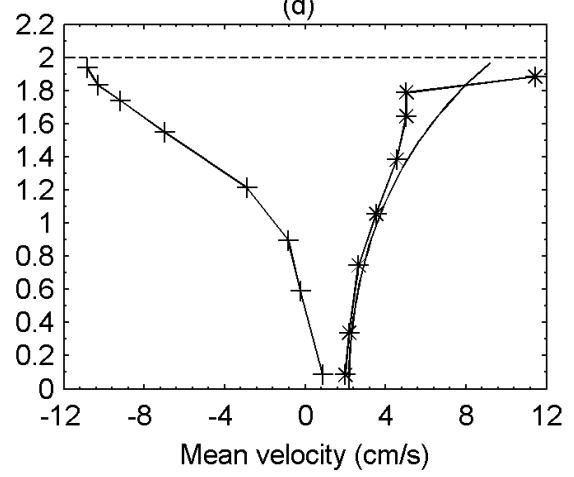

Figure 9. Trend of the Stokes drift and of the mean velocity in the first station for (a) $\mathrm{H}=0.5 \mathrm{~m}, \mathrm{~T}=5.5 \mathrm{~s}$ (b) $\mathrm{H}=0.5 \mathrm{~m}, \mathrm{~T}=3 \mathrm{~s}$ (c) $\mathrm{H}=0.6, \mathrm{~T}=5.5 \mathrm{~s}$ (d) $\mathrm{H}=0.6 \mathrm{~m}, \mathrm{~T}=3 \mathrm{~s}$. Line with crosses: Eulerian mean velocity; line with stars: experimental Stokes drift; line: theoretical Stokes drift computed by the formula valid for waves propagating over a constant depth.

Preliminarily, it is interesting to observe that for $T=5.5 \mathrm{~s}$ the theoretical Stokes drift shows the same qualitative behavior of the experimental one, while for $T=3 \mathrm{~s}$ the good agreement appears to be also quantitative, apart close to the trough. It can be observed that for large periods, i.e. for waves in shallow water, the mean Eulerian velocity is far to be the mirror image of the Stokes drift as required by Eq. 4. Results closer to those predicted by the previous equation are attained for $T=3 \mathrm{~s}$, i.e. for larger values of $k h$.

The existence in literature of experimental measurements in which the mean velocity shows a behaviour opposite to the Stokes drift, highlight that the present mean velocity profiles are not alone to exhibit a convexity turned towards offshore. Indeed, Swan (1990) reported that during the initial stages of his experiments the mean velocity profiles were similar to those predicted by the diffusion solution but in the following they did not tend to the theoretical profiles but just to their opposite. The author hypothesized that this results could be due to the vorticity generated by the breaking and advected offshore by the mean velocity.

\section{Conclusions}

In this paper results about the experimental measurements of the mean velocity induced by sea waves offshore the breaker line have been presented and discussed. Such measurements have been carried out in a large wave-flume in order to minimize the scale effects. The measurements show that the convexity of the mean velocity profiles is turned towards offshore. Such result is opposite both to that predicted by the theory and to that mainly reported by other experimental measurements. However, it has been highlighted that experimental measurements are reported in literature which are in agreement with the present ones.

The reasons why in the experiments the mean velocity distribution is different from that reported in other experiments is not well known and it requires further close examination of the phenomenon of the steady current in order to be clarified.

In the present experiments it has been observed that close to the wave trough the mean velocity is directed offshore and increases in this direction when the period decreases. Close to the bottom we observed just the opposite, indeed here the velocity is in general offshore directed and increases in this 
direction when the wave period increases. A consequence of this behavior is that for large periods the mean velocity tends to be more constant along the water depth.

Near to the bottom, the presence of a mean velocity directed offshore, that is opposite to what expected according to the theory, has been explained by taking into account that the bottom boundary layer is turbulent and the waves are asymmetric. Indeed, previous studies have shown that in this case the turbulence produces a time mean Reynolds stress which drives a steady streaming directed offshore. We showed that the velocity increases in the offshore direction as the wave asymmetry increases, however, for small asymmetry the mean velocity can be onshore directed.

When the relative water depth $k h$ increases the mean velocity seems to tend towards values which are the opposite of the Stokes drift as it has recently been shown in literature. A more accurate check of this tendency requires further experiments with waves characterized by shorter periods with respect to those here considered.

\section{ACKNOWLEDGMENTS}

This work has been supported by European Community's Sixth Framework Program through the grant to the budget of the Integrated Infrastructure Initiative HYDRALAB III within the Transnational Access Activities, Contract no. 022441. Thanks are due to PRIN 2008 project 'Operative instruments for the estimate of coastal vulnerability in the presence of sandy beaches and also in the presence of coastal structures'.

\section{REFERENCES}

Bagnold, R.A. 1947. Sand movement by waves: some small scale experiments with sand of very low density, J. Inst. Civil Engrs., 27,447-469.

Dean, R.G., and R.A. Dalrymple. 1993. Water waves mechanics for engineers and scientists, World Scientific.

Holmedal, L.E., and D. Myrhaugh. 2009. Wave-induced steady streaming, mass transport and net sediment transport in rough turbulent ocean bottom boundary layers, Cont. Shelf Res., 29, 911-926.

Hwung, H.H., and C. Lin. 1990. The mass transport of waves propagating on a sloping bottom, In Proc. $22^{\text {nd }}$ Int. Conf. Coastal Engineering, ASCE, 544-556, Reston.

Longuet-Higgins, M.S. 1953. Mass transport in water waves, Proc. R. Soc. A, 245, 535-581.

Monismith, S.G., Cowen, E.A., Nepf, H.M., Magnaudet, J., and L. Thais. 2007. Laboratory observations of mean flows under surface gravity waves, J. Fluid Mech., 573, 131-147.

Nadaoka, K., and T. Kondoh. 1982. Laboratory measurements of velocity field structure in the surf zone by LDV, Coast. Eng. Japan, 25, 125-145.

Putrevu, U., and I. Svendsen. 1993. Vertical structure of the undertow outside the surf zone, $J$. Geophys. Res. Oceans, 98(C12).

Russel, R.C.H., and J.D.C. Osorio. 1958. An experimental investigation of drift profiles in a closed channel, In Proc. $6^{\text {th }}$ Coastal Engng Conf., ASCE, 171.

Scandura, P. 2007. Steady streaming in a turbulent oscillating boundary layer, J. Fluid Mech., 571, 265280.

Sleath, J.F.A. 1984. Measurements of mass transport over a rough bed, In Proc. $19^{\text {th }}$ Coastal Engng C27, 1149-1160.

Stokes, G.G. 1847. On the theory of oscillatory waves, Trans. Camb. Phil. Soc., 8, 441-455.

Swan, C. 1990. Convection within an experimental wave flume, J. Hydr. Res, 28(3), 273-282. 